\title{
Stochastic Discounting in Repeated Games: Awaiting the Almost Inevitable*
}

\author{
Mehmet Barlo ${ }^{\dagger} \quad$ Can Ürgün ${ }^{\ddagger}$
}

August, 2011

\begin{abstract}
We study repeated games with pure strategies and stochastic discounting under perfect information, with the requirement that the stage game has at least one pure Nash action profile. Players discount future payoffs with a common, but stochastic, discount factor where associated stochastic discounting processes are required to satisfy Markov property, martingale property, having bounded increments, and possessing state spaces with rich ergodic subsets. We, additionally, demand that there are states resulting in discount factors arbitrarily close to 0 , and that they are reachable with positive (yet, possibly arbitrarily small) probability in the long run. In this setting, we prove both the perfect Folk Theorem and our main result: The occurrence of any finite number of consecutive repetitions of the period Nash action profile, must almost surely happen within a finite time window no matter which subgame perfect equilibrium strategy is considered and no matter how high the initial discount factor is.
\end{abstract}

Journal of Economic Literature Classification Numbers: C72; C73; C79

Keywords: Repeated Games; Stochastic Discounting; Stochastic Games; Folk Theorem; Stopping Time

*This is a revised version of Ürgün (2011). We thank Ahmet Alkan, Uluğ Çapar, Guilherme Carmona, Alpay Filiztekin, Hülya Eraslan, Maarten Janssen, Thomas Jungbauer, Ehud Kalai, Özgür Kıbrıs, Han Özsöylev, and participants at the Micro Research Seminar at the Vienna Graduate School of Economics and the Economics Seminar at the Sabancı University, the NASM 2011 conference, the 2011 meeting of EWGET for helpful comments and suggestions. Any remaining errors are ours.

${ }^{\dagger}$ Corresponding Author: FASS, Sabancı University, Orhanll, Tuzla, 34956, Istanbul, Turkey; Phone: +90 216483 9284; Fax: +90 2164839250 (CC. M. Barlo); email: barlo@sabanciuniv.edu.

${ }^{\ddagger}$ Sabancı University, Orhanlı, Tuzla, 34956, Istanbul, Turkey; email: curgun@sabanciuniv.edu; and Department of Managerial Economics and Decision Sciences, Kellogg School of Management, Northwestern University, Evanston, IL 60208, USA; email: c-urgun@kellogg.northwestern.edu 


\section{Introduction}

The Folk Theorems of Aumann and Shapley (1994) and Fudenberg and Maskin (1986) establish that payoffs which can be approximated in equilibrium with patient players, are equal to the set of individually rational ones. The main reason for this observation is players' ability to coordinate their actions using past behavior. In turn, this vast multiplicity of equilibrium payoffs, considerably weakens the predictive power of game theoretic analysis. Moreover, the consideration of limited memory and bounded rationality, lack of perfect observability of the other players' behavior and the past, and uncertainty of future payoffs do not change this conclusion ${ }^{1}$ An important aspect of all these findings is the use of constant discounting. ${ }^{2}$ On the other hand, allowing for the discount factor to depend on the history of the game and/or vary across time, is not extensively analyzed in the literature on repeated games. ${ }^{3}$

The current paper studies repeated games with pure strategies and common stochastic discounting under perfect information with the requirement that the stage game is any finite normal form game possessing at least one pure Nash action profile. Players are assumed to discount future payoffs with a common, but stochastic, discount factor. Each player observes the current one-shot discount factor before making a choice of action in that period. Moreover, the associated stochastic discounting processes are required to satisfy the following: (1) Markov property; (2)

\footnotetext{
${ }^{1}$ These observations are documented in various studies including Kalai and Stanford (1988), Sabourian (1998), Barlo, Carmona, and Sabourian (2009), Barlo, Carmona, and Sabourian (2007); Fudenberg, Levine, and Maskin (1994), Hörner and Olszewski (2006), Mailath and Olszewski (2011); Dutta (1995), Fudenberg and Yamamato (2010), and Hörner, Sugaya, Takahashi, and Vieille (2009).

${ }^{2} \mathrm{An}$ accepted interpretation of the use of discounting in repeated games, offered by Rubinstein (1982) and Osborne and Rubinstein (1994), is that the discount factor determines the probability of the strategic interaction surviving into the next period. Thus, constant discounting implies that this probability is independent of the history of the game, in particular, invariant.

${ }^{3}$ There is a vast body of related work concerning stochastic interest rates that can be found in the theory of finance. To that regard, we refer the reader to Ross (1976), Harrison and Kreps (1979), and Hansen and Richard (1987).
} 
martingale property; (3) have bounded increments (across time) and possess state spaces with rich ergodic subsets; (4) there are states of the stochastic discounting process that are arbitrarily close to 0 , and such states can be reached with positive (yet, possibly arbitrarily small) probability in the long run.

In this setting, we, not only establish the subgame perfect Folk Theorem, but also prove the main result of this study, the inevitability of Nash behavior: The occurrence of any finite number of consecutive repetitions of the period Nash action profile, must almost surely happen within a finite time window no matter which subgame perfect equilibrium strategy is considered and no matter how high the initial level of the stochastic discounting process is. In other words, for all levels of the initial oneshot discount factor every equilibrium strategy profile must almost surely involve a stage, i.e. each stochastic process governing the one-shot discount factors possesses a stopping time, after which long consecutive repetitions of the period Nash action profile must be observed. 4

The fundamental reason of our main result is captured by a significant phrase to be found on page 101 of Williams (1991): "Whatever always stands a reasonable chance of happening, will almost surely happen - sooner rather than later." Indeed, we prove that for any $\varepsilon>0$ and for any given initial level of the stochastic discounting process, the one-shot discount factor must almost surely fall below $\varepsilon$ in a finite time period. Then, given any natural number $K$, the restriction of bounded increments enables us to identify the desired level of $\varepsilon$ so that the one-shot discount factors cannot exceed a certain threshold even when $K+1$ consecutive "good" shocks are realized.

It needs to be emphasized that the inevitability of Nash behavior, an event that

\footnotetext{
${ }^{4}$ Considering the repeated prisoners' dilemma with stochastic discounting, our results display that: (1) the subgame perfect Folk Theorem holds; and, (2) in any subgame perfect equilibrium strategy for any natural number $K$, the occurrence of $K$ consecutive defection action profiles must happen almost surely within a finite time period, regardless of the initial level of the stochastic one-shot discount factor.
} 
almost surely happens in some distant future, should not be interpreted as an "AntiFolk Theorem". In fact, the punchline of this study is that both of the inevitability of Nash behavior and the subgame perfect Folk Theorem results hold in a wide class of repeated games with stochastic discounting. This, in turn, establishes the following observation: When the initial level of the stochastic discounting process is high enough, the inevitability result does not have a sufficiently strong impact on the state contingent and time consistent plans of actions drafted and evaluated with the information available at date zero.

In order to explain why the subgame perfect Folk Theorem holds in this environment, first, it needs to be pointed out that our requirements on stochastic discounting processes imply the evaluation of payoffs in repeated games with constant discounting being closely related to that with stochastic discounting. Given any repeated game under perfect information and a constant discount factor $\hat{\delta} \in(0,1)$, a repeated game under perfect information and stochastic discounting with the initial level of the stochastic one-shot discount factor equalling $\hat{\delta}$, can be interpreted as a perturbation of the original game, and it exhibits the following properties: (1) The date zero expectations of the stochastic one-shot discount factors are all equal to $\hat{\delta}$; and (2) with date zero expectations, players employ weaker discounting than that associated with a constant and common discount factor $\hat{\delta}$; and (3) at any history, expected level of future one-shot discount factors are equal to the current one.

Using this observation, we prove that given any history of shocks, players evaluate future payoffs of a given strategy profile with expectations of future one-shot discount factors, where these expectations are formed with information given by the history of shocks. That is, we prove that the conclusions of Abreu (1988) and Abreu, Pearce, and Stachetti (1990) apply: Given any time period $t$ and any history of shocks up to period $t$, the set of subgame perfect continuation payoffs is compact. Thus, when checking whether or not a given strategy profile is subgame perfect, the following suffices: For any period $t$ and any history of shocks and actions up to $t$, the continuation utility of 
every player must exceed the one given by a player deviating singly (from the action profile prescribed by the strategy for that particular history) followed by him being punished in the most severe and credible manner for the rest of the game. It needs to be emphasized that, the specific form of a player's punishment, triggered by him deviating singly in period $t$, also depends on the particular realizations of the oneshot discount factors in period $t+1$. This is because, we require the punishment to be subgame perfect, thus, optimal in the subgame that starts in period $t+1$ where players observe the current level of the one-shot discount factor before choosing actions.

Having established that the check for subgame perfection reduces to the comparison of the continuation payoffs obtained by conforming with those delivered by deviation followed by punishment, for any player and for any history of actions and shocks, we identify a class of strategies with which this task becomes manageable. Indeed, this kind of strategies also help in the construction of an analogy between repeated games with stochastic discounting and those with constant discounting. We concentrate on strictly enforceable strategies in repeated games with constant discounting: 5 Those, for which the incentive inequalities hold strictly with a strictly positive slack independent from the identity of the player and the date. Clearly, such strategies are subgame perfect in the repeated game with constant discounting. Then, for a given strictly enforceable strategy in a repeated game with constant discounting $\hat{\delta}$, we construct an extension of it in the repeated game with stochastic discounting with the initial level given by $\hat{\delta}$, as follows: It prescribes the play to continue as dictated by its counterpart in the repeated game with constant discounting, whenever each of the past realizations of the one-shot discount factors exceeds a date and state specific threshold. Otherwise, our strategy recommends the play to consist of the repetitions of a Nash action profile of the stage game thereafter. Then, we show that, when the initial level of the stochastic discounting process, $\hat{\delta}$, is sufficiently high, we

\footnotetext{
${ }^{5}$ This notion is first used in Barlo, Carmona, and Sabourian (2009), and is closely related to patient strictness of Mailath and Olszewski (2011).
} 
can construct date and state specific thresholds such that, given a date and state, the probability (evaluated in that date and state) of the one-shot discount factor of the next period falling below its associated threshold, is sufficiently low. This, in turn, implies that when the initial level of the stochastic discounting process is chosen sufficiently high, our extension -of the strictly enforceable strategy in the repeated game with constant discounting- is subgame perfect. Meanwhile, because that $\hat{\delta}$ is sufficiently high, the associated date-zero utility profile of the extension is arbitrarily close to that of its counterpart in the repeated game with constant discounting.

Finally, the subgame perfect Folk Theorem for repeated games with stochastic discounting is obtained by combining the above construction and the observation that when restricted to pure actions, the strategy profile in the proof of the subgame perfect Folk Theorem of Fudenberg and Maskin (1991) is, in fact, strictly enforceable.

The literature on stochastic discounting in repeated games is surprisingly not rich. A significant contribution is Baye and Jansen (1996). This study considers a form of stochastic discounting with no stringent restrictions on the values that oneshot discount factors can take, and the distributions of one-shot discount factors may depend on the time index. However, in contrast to ours, their setting does not involve history dependent stochastic discounting. Moreover, they identify two significant cases: The first, when the one-shot discount factor is realized before the actions in the stage game are undertaken; the second, when the actions need to be chosen before the one-shot discount factor is realized. They prove that the Folk Theorem holds with in the latter case. However, they show that in the former case the "full" Folk Theorem "...breaks down; payoffs on the boundary of the set of individually rational payoffs are unobtainable as Nash equilibrium average payoffs to the supergame." In fact, our formulation, at first sight, seems to correspond to their second case because our setting involves the one-shot discount factor being common knowledge before actions are chosen. Consequently, our "full" Folk Theorem may appear to be at odds with their findings. However, there is a critical difference which concerns the 
beginning of the game. In our model, the initial level of the one-shot discount factor is deterministic and common knowledge. Indeed, the failure of the "full" Folk Theorem shown in the second setting of Baye and Jansen (1996) is primarily due to the action profile chosen in date-zero being a function of the random period-zero discount factor observed by players before the date-zero action is chosen.

There is a number of notable contributions in the context of Folk Theorems in stochastic games. Indeed, recent significant studies by Fudenberg and Yamamato (2010) and Hörner, Sugaya, Takahashi, and Vieille (2009) generalize the Folk Theorem of Dutta (1995) for irreducible stochastic games with the requirement of a finite state space. In fact, our setup can be expressed as an irreducible stochastic game where discounting is constant, yet the stochastic stage game payoffs are obtained using a single stochastic scalar, and the actions chosen have no bearing on future payoffs. Indeed, even though the punchline of our study is not just the Folk Theorem, it needs to be mentioned that the repeated games with stochastic discounting that the current study concentrates on, are particular irreducible stochastic games, however, with infinite state spaces. Hence, none of these significant Folk Theorems apply.

The organization of the paper is as follows: The next section will present the preliminaries. In section 3, we characterize the set of subgame perfect equilibrium payoffs. In section 4, we present and prove the main theorem of this study. Finally, in section 5, the subgame perfect Folk Theorem for repeated games with stochastic discounting is stated and proven.

\section{Preliminaries}

Let $G=\left(N,\left(A_{i}, u_{i}\right)_{i \in N}\right)$ be a normal form game with $|N| \in \mathbb{N}$ and for all $i \in N, A_{i}$ is player $i$ 's actions with property that $\left|A_{i}\right| \in \mathbb{N}$; and $i$ 's payoff function denoted by $u_{i}: A \rightarrow \mathbb{R}$ where $A=\prod_{i \in N} A_{i}$ and $A_{-i}=\prod_{j \neq i} A_{i}$.

For what follows, we assume that the stage game, $G$, has a pure strategy Nash 
equilibrium:

Assumption $1 G=\left(N,\left(A_{i}, u_{i}\right)_{i \in N}\right)$ is such that there exists $a^{*} \in A$ with the property that for all $i \in N, u_{i}\left(a^{*}\right) \geq u_{i}\left(a_{i}, a_{-i}^{*}\right)$ for all $a_{i} \in A_{i}$.

For any $i \in N$ denote the (pure strategy) minmax payoff and a (pure strategy) minmax profile for player $i$ by $v_{i}=\min _{a_{-i} \in A_{-i}} \max _{a_{i} \in A_{i}} u_{i}\left(a_{i}, a_{-i}\right)$ and the associated action profile by $m^{i} \in \arg \min _{a_{-i} \in A_{-i}} \max _{a_{i} \in A_{i}} u_{i}\left(a_{i}, a_{-i}\right)$, respectively. The set of individually rational payoffs is denoted by $\mathcal{U}=\left\{u \in \operatorname{co}(u(A)): u_{i} \geq v_{i}\right.$ for all $\left.i \in N\right\}$, and the set of strictly individually rational payoffs by $\mathcal{U}^{0}=\left\{u \in \operatorname{co}\left(u(A): u_{i}>\right.\right.$ $v_{i}$ for all $\left.i \in N\right\}$.

The supergame of $G$ consists of an infinite sequence of repetitions of $G$ taking place in periods $t=0,1,2,3, \ldots$ Let $\mathbb{N}_{0}=\mathbb{N} \cup\{0\}$.

In every period $t \in \mathbb{N}_{0}$, a random variable, $\mathbf{d}_{t}$, is determined, forming a stochastic process $\left\{\mathbf{d}_{t}\right\}_{t \in \mathbb{N}_{0}}$. The following summarizes the assumptions needed, which allows for a wide class of stochastic processes:

Assumption $2\left\{\mathbf{d}_{t}\right\}_{t \in \mathbb{N}_{0}}$ is a stochastic process satisfying the following:

1. Markov property;

2. martingale property;

3. the state space $\Omega$ of $\left\{\mathbf{d}_{t}\right\}_{t}$, is a subset of $(0,1)$;

4. given $\Omega$, the set of ergodic states, $\Omega^{E}$, is dense in $\Omega$;

5. for any $\varepsilon>0$, there exists $\tau \geq t$ with $\operatorname{Pr}\left[\mathbf{d}_{\tau}<\varepsilon \mid \mathcal{F}_{t}\right]>0$;

6. for any given state $\omega \in \Omega \subseteq(0,1)$, the set of states $\omega^{\prime} \in \Omega$ that are reachable from $\omega$ in a single period and satisfying $\omega<\omega^{\prime}$, denoted by $R(\omega)$, is finite. Moreover, for any $\omega, \omega^{\prime} \in \Omega$ with $\omega^{\prime} \geq \omega, \sup R\left(\omega^{\prime}\right) \geq \sup R(\omega)$;

7. $\mathbf{d}_{0}$ is non-stochastic. 
The first two parts of Assumption 2 imply that expectations about the future depend only on the current value of the stochastic process, and are equal to the current value. The third and fourth parts of Assumption 2 imply that the set of values reachable is $(0,1)$, and the set of aperiodic and non-transient states must be dense in the state space. In the fifth part of Assumption 2, we require that there are states arbitrarily close to 0 , and such states can be reached with positive, but possibly arbitrarily small, probability in the long run. It is essential to note that when the state space of the stochastic process is finite, then the fifth part of our assumption cannot hold. The sixth part of Assumption 2 requires that the "upward jumps" in the process cannot involve infinitely many states. Indeed, it can be viewed as a special form of the standard bounded increments requirement, which is satisfied due to this process being bounded. In other words, the above requirement limits the increments to be bounded non-trivially at every state. The final part of Assumption 2 requires that the initial level of the stochastic process is deterministic.

We wish to point out that the stochastic process known as the normalized betabinomial distribution with two dimensions, a Polya's urn scheme $\frac{6}{6}$ satisfies all the requirements of Assumption 2, where the relevant state space $\Omega$ is a subset of rational numbers in $(0,1){ }^{7}$ For more specifics, we refer the reader to Karlin and Taylor (1975).

${ }^{6}$ Define $\left\{\mathbf{d}_{t}\right\}_{t}$ as follows: Without loss of generality, let $\mathbf{d}_{0}=\hat{\delta}$ be a rational number in $(0,1)$. Thence, $\hat{\delta}=\frac{g}{g+b}$ for some $g, b \in \mathbb{N}$, where $g$ is interpreted as the number of "good", $b$ as the "bad", balls in the urn. A ball is drawn randomly, and is put back into the urn along with a new ball of the same nature, and this process is repeated in each round. Thus, the support of $\mathbf{d}_{1}$ is $\left\{\frac{g+1}{g+1+b}, \frac{g}{g+1+b}\right\}$ where the first observation happens with probability $\mathbf{d}_{0}$. Inductively, for any $t>1$ given $d_{t-1}$ (a realization of $\mathbf{d}_{t-1}$ ) the support of $\mathbf{d}_{t}$ equals $\left\{\frac{g+k+1}{g+b+t}, \frac{g+k}{g+b+t}\right\}$ where $k \leq t$ denotes the number of good balls drawn up to period $t$ and the first element of this support is drawn with a probability given by $d_{t-1}$.

${ }^{7}$ In some sources, the Polya scheme is defined directly by the rational number obtained from the ratio (of the number of good balls over the number of total balls). Such definitions do not distinguish between having 1 good ball among 2 and having 50 good balls among 100. Consequently, the Markov property does not hold when such a definition is employed. On the other hand, the same stochastic 
Given a stochastic process $\left\{\mathbf{d}_{t}\right\}_{t \in \mathbb{N}_{0}}$, let $\left\{\mathcal{F}_{t}\right\}_{t \in \mathbb{N}_{0}}$ be a filtration (i.e. sequence of growing $\sigma$-algebras); and, for any given $t \in \mathbb{N}_{0}, \mathcal{F}_{t}$ is commonly interpreted as the information in period $t$.

Given $\tau$, we let a particular realization of the stochastic process $\left\{\mathbf{d}_{t}\right\}_{t \in \mathbb{N}_{0}}$ be denoted by $d_{\tau} \in \mathbb{R}$.

The supergame is defined for a given $\left\{\mathbf{d}_{t}\right\}_{t}$ with $\hat{\delta}=r \mathbf{d}_{0}$ and $r \in(0,1]$, and is denoted by $G\left(\left\{\mathbf{d}_{t}\right\}_{t}\right) . \stackrel{8}{ }$ For $k \geq 1$, a $k$-stage history is a $k$-length sequence $h_{k}=$ $\left(\left(a_{0}, d_{1}\right), \ldots,\left(a_{k-1}, d_{k}\right)\right)$, where, for all $0 \leq t \leq k-1, a_{t} \in A$; and for all $1 \leq t \leq k$, $d_{t}$ is realization of $\mathbf{d}_{t}$; the space of all $k$-length histories is $H_{k}$, i.e., $H_{k}=(A \times \mathbb{R})^{k}$. We use $e$ for the unique 0 -stage history - it is a 0-length history that represents the beginning of the supergame. The set of all histories is defined by $H=\bigcup_{n=0}^{\infty} H_{n}$. For every $h \in H$, we let $\ell(h)$ denote the length of $h$. For $t \geq 2$, we let $d^{t}=\left(d_{1}, \ldots, d_{t}\right)$ denote the history of shocks up to and including period $t$.

We assume that players have complete information. That is, in period $t>0$, knowing the history up to period $t$, given by $h_{t}$, the players make simultaneous moves denoted by $a_{t, i} \in A_{i}$. The players' choices in the unique 0 -length history $e$ are in $A$ as well. Notice that in our setting, given $t$, a player not only observes all the previous action profiles, but also all the shocks including the ones realized in period $t$. In other words, the period- $t$ shocks are commonly observed before making a choice in period process can be defined by the number of good balls divided by the total number of balls, where the information kept consists of the number of good balls and the number of total balls. Then, the process is a Markovian martingale. To see this, observe that a stochastic process defined by the number of good balls is clearly Markovian, and it is a martingale with respect to 1 divided by the number of total balls.

${ }^{8}$ The reason why we have chosen to formulate $\hat{\delta} \in(0,1)$ as a multiplication of a real number $r$ in $(0,1]$ and $\mathbf{d}_{0}$ is as follows: The stochastic process at hand may involve states spaces that are strict subsets of $(0,1)$. As an example consider the stochastic process given by Polya's urn, defined in footnote 6 . Then, the state space is a subset of the rational numbers in $(0,1)$. Hence, for obtaining $\hat{\delta}$ precisely, a multiplication with a real number in $(0,1]$ might be necessary, when $\hat{\delta}$ is not a rational number. 
$t$.

For all $i \in N$, a strategy for player $i$ is a function $f_{i}: H \rightarrow A_{i}$ mapping histories into actions. The set of player $i$ 's strategies is denoted by $F_{i}$, and $F=\prod_{i \in N} F_{i}$ is the joint strategy space. Finally, a strategy vector is $f=\left(f_{1}, \ldots, f_{n}\right)$. Given an individual strategy $f_{i} \in F_{i}$ and a history $h \in H$ we denote the individual strategy induced at $h$ by $f_{i} \mid h$. This strategy is defined point-wise on $H:\left(f_{i} \mid h\right)(\bar{h})=f_{i}(h \cdot \bar{h})$, for every $\bar{h} \in H$. We will use $(f \mid h)$ to denote $\left(f_{1}\left|h, \ldots, f_{n}\right| h\right)$ for every $f \in F$ and $h \in H$. We let $F_{i}\left(f_{i}\right)=\left\{f_{i} \mid h: h \in H\right\}$ and $F(f)=\{f \mid h: h \in H\}$.

A strategy $f \in F$ induces an outcome $\pi(f)$ as follows: $\pi^{0}(f)=f(e) \in A$; and for $d_{1} \in \mathbb{R}$ we have $\pi^{1}(f)\left(d^{1}\right)=f\left(f(e), d_{1}\right) \in A ;$ and, $\pi^{2}(f)\left(d^{2}\right)=f\left(f(e), f\left(f(e), d_{1}\right), d_{2}\right) \in$ $A, d_{1}, d_{2} \in \mathbb{R} ;$ and continuing in this fashion we obtain

$\pi^{k}(f)\left(d^{k}\right)=f\left(\pi^{0}(f), \pi^{1}(f)\left(d^{1}\right), \ldots, \pi^{k-1}(f)\left(d^{k-1}\right), d_{k}\right) \in A, k>1$ and $d_{1}, \ldots, d_{k} \in \mathbb{R}$.

On the other hand, the repeated game with common and constant discounting, with a discount factor $\hat{\delta} \in(0,1)$, is denoted by $\bar{G}(\hat{\delta})$. We employ the above definitions without the parts concerning the stochastic discounting process.

Next, we wish to present the construction of expected payoffs. Due to that regard, first we will present our stochastic discounting construction, and second formulate the resulting expected utilities.

Players payoffs are evaluated with a common stochastic discount factor, denoted by $\left\{\mathbf{d}_{t}^{t+1}\right\}_{t \in \mathbb{N}_{0}}$, where for any given $t \in \mathbb{N}_{0}, \mathbf{d}_{t}^{t+1}$ identifies the probability of the game continuing from period $t$ to period $t+1$. Hence, the stochastic discount factor from period $t$ to period $\tau$, with $\tau \geq t+1$, is defined by $\mathbf{d}_{t}^{\tau} \equiv r \prod_{k=t}^{\tau-1} \mathbf{d}_{k}$, for some $r \in(0,1]$ with the convention that $\mathbf{d}_{t}^{t}=1$. This trivially implies that $\mathbf{d}_{t}^{t+1}=r \mathbf{d}_{t}$. We often denote $\mathrm{E}\left(\mathbf{d}_{t}^{\tau} \mid \mathcal{F}_{s}\right)$ by $\mathrm{E}_{s}\left(\mathbf{d}_{t}^{\tau}\right), s \leq t \leq \tau$. For any $t \in \mathbb{N}_{0}$, we let a realization of $\mathbf{d}_{t}^{t+1}$ be denoted by $\delta_{t}^{t+1}$, which stands for the realized probability that the game continues from period $t$ to period $t+1$. In the rest of the paper, we often abuse notation and denote $\mathrm{E}\left(\mathbf{d}_{t}^{\tau} \mid \mathcal{F}_{s}\right)$ (i.e. $\left.\mathrm{E}_{s}\left(\mathbf{d}_{t}^{\tau}\right)\right)$ by $\mathrm{E}\left(\delta_{t}^{\tau} \mid \mathcal{F}_{s}\right)$ and $\mathrm{E}_{s}\left(\delta_{t}^{\tau}\right)$, for $s \leq t \leq \tau$. 
One thing to note is the particular timing and information setting that we employ: Given $r \mathbf{d}_{0}=\hat{\delta}$ the stochastic discount factor determining the probability that the game continues into the next period is pinned down to a constant, $\mathbf{d}_{0}^{1}=r \mathbf{d}_{0}=\hat{\delta}$. In the next period, $t=1, \mathbf{d}_{1}$ is realized before players decide on $a_{1} \in A$. So, the realization of $r \mathbf{d}_{1}=\mathbf{d}_{1}^{2}$ is also known at $t=1$. Thus, following an inductive argument in any period $t>1$, the given $d^{t}$ determines the particular level of $\delta_{t}^{t+1}$.

The following Lemma displays that the stochastic discounting process constructed in this study involves weaker discounting than the one associated with constant discounting:

Lemma 1 Suppose that Assumption 2 is satisfied. Then

1. every possible realization of $\mathbf{d}_{t}^{\tau}$ is in $(0,1)$ for every $\tau, t \in \mathbb{N}_{0}$ with $\tau>t$,

2. $\mathrm{E}\left(\mathbf{d}_{t}^{t+1} \mid \mathcal{F}_{0}\right)=\delta_{(0)}$ for some $\delta_{(0)} \in(0,1)$ and for all $t \in \mathbb{N}_{0}$,

3. for every given $\hat{\delta} \in(0,1)$, there exists $r \in(0,1]$ such that $\delta_{(0)}=\hat{\delta}$,

4. for every $\tau, t, s \in \mathbb{N}_{0}$ with $\tau>s \geq t$, given $\mathbf{d}_{t}^{t+1}=\delta_{t}^{t+1}$

$$
\mathrm{E}\left(\mathbf{d}_{\tau}^{\tau+1} \mid \mathcal{F}_{t}\right)=\delta_{t}^{t+1}
$$

and

$$
\mathrm{E}\left(\mathbf{d}_{s}^{\tau} \mid \mathcal{F}_{t}\right) \geq\left(\mathrm{E}\left(\mathbf{d}_{s}^{s+1} \mid \mathcal{F}_{t}\right)\right)^{\tau-s}=\left(\delta_{t}^{t+1}\right)^{\tau-s}
$$

The implications of this Lemma are essential for the proof, the interpretation and the evaluation of our results:

The first one displays that the stochastic process specified results in a well-defined construction for stochastic discounting. This is because for every $\tau, t \in \mathbb{N}_{0}$ with $\tau>t$, $\mathbf{d}_{t}^{\tau}=r \prod_{k=t}^{\tau-1} \mathbf{d}_{k}$ is in $(0,1)$ which is due to $r \in(0,1]$, and every possible realization of $\mathbf{d}_{k}$ for every $k \in \mathbb{N}_{0}$ being in $(0,1)$.

The second shows that date zero expectations of future one-period discount factors are constant with respect to the time index. 
And the third, displays that $r$ can be chosen so that any given constant discount factor can be precisely obtained. In fact, we wish to point out that the reason for using a real number $r \in(0,1]$ in the definition given by $\mathbf{d}_{t}^{\tau} \equiv r \prod_{k=t}^{\tau-1} \mathbf{d}_{k}$ (and not simply letting $r=1$ ) is that our construction does not necessarily require the stochastic processes to have a support consisting the entirety of $(0,1)$. Therefore, when dealing with stochastic processes requiring $\Omega \neq(0,1)$, for any $\hat{\delta} \in(0,1) \backslash \Omega, r \in(0,1]$ and $\hat{\omega} \in \Omega$ can be identified such that $r$ is sufficiently close to 1 and $\hat{\delta}=r \hat{\omega}$. Thus, without loss of generality, we assume $r=1$ in the rest of this study.

Using the first three results presented in the above Lemma, we conclude with respect to date zero expectations, the repeated game at hand can be associated with one having a constant and common discount factor. Thus, our repeated game with stochastic discounting can be interpreted as a perturbation of a "standard" repeated game under perfect information with common and constant discount factor.

Finally, the fourth implication of Lemma 1 is twofold: Given any history of shocks up to time period $t$, the first implication is that the expected level of future oneshot discount factors are equal to the current one. The second shows that every player values future returns more than a player using a constant discount factor obtained from the same shocks. That is, a player discounts a return in period $\tau$, $\tau>t$, with $\mathrm{E}_{t}\left(\mathbf{d}_{t}^{\tau}\right)$ which is greater or equal to $\left(\mathrm{E}_{t}\left(\mathbf{d}_{t}^{t+1}\right)\right)^{\tau-t}$. (Notice that given $d^{t}$, $\mathrm{E}_{t}\left(\mathbf{d}_{t}^{\tau}\right)=\delta_{t}^{t+1} \mathrm{E}_{t}\left(\mathbf{d}_{t+1}^{\tau}\right)$, because $\mathbf{d}_{t}^{t+1}=\delta_{t}^{t+1}$ is realized.) In particular, this implies $\hat{\delta}$ can be chosen so that

$$
\mathrm{E}_{0}\left(\mathbf{d}_{t}^{\tau}\right) \geq\left(\mathrm{E}_{0}\left(\mathbf{d}_{t}^{t+1}\right)\right)^{\tau-t}=\hat{\delta}^{\tau-t}=\left(\delta_{(0)}\right)^{\tau-t}
$$

and when $\tau=t+1$ then this inequality holds with an equality. Hence, these properties establish that with a date 0 point of view, our stochastic discounting construction involves weaker discounting than that associated with a constant and common discount factor.

Proof of Lemma 1. The proofs of parts 1,2 and 3 of the Lemma are already discussed above. The first part of the fourth result is, in fact, the martingale identity. 
For the second part, notice that

$$
\begin{aligned}
\mathrm{E}\left(\mathbf{d}_{s}^{\tau} \mid \mathcal{F}_{t}\right) & =r \mathrm{E}\left(\prod_{k=s}^{\tau} \mathbf{d}_{k} \mid \mathcal{F}_{t}\right)=r \mathrm{E}\left(\mathbf{d}_{s} \mathrm{E}\left(\mathbf{d}_{s+1} \ldots \mathrm{E}\left(\mathbf{d}_{\tau-1} \mid \mathcal{F}_{\tau-1}\right) \ldots \mid \mathcal{F}_{s}\right) \mid \mathcal{F}_{t}\right) \\
& \geq r \mathrm{E}\left(\left(\mathbf{d}_{s}\right)^{(\tau-s)} \mid \mathcal{F}_{t}\right) \geq\left(\mathrm{E}\left(\mathbf{d}_{s}^{s+1} \mid \mathcal{F}_{t}\right)\right)^{\tau-s}=\left(\mathrm{E}\left(\delta_{t}^{t+1} \mid \mathcal{F}_{t}\right)\right)^{\tau-s}
\end{aligned}
$$

due to the tower property (see Williams (1991)), the martingale identity and Jensen's inequality.

The next Assumption is about how players employ knowledge of the past when taking expectations:

Assumption 3 In every period $t \in \mathbb{N}_{0}$, each player uses the most up to date information, i.e. $\mathcal{F}_{t}$.

Given a strategy profile $f$, because that each period's supremum return is bounded for every player, the payoff of player $i \in N$ in the supergame $G\left(\left\{\mathbf{d}_{t}\right\}_{t}\right)$ of $G$ is, where $\mathbf{d}_{0}=\hat{\delta} \in(0,1)$ :

$$
\begin{aligned}
U_{i}\left(f,\left\{\mathbf{d}_{t}\right\}_{t}\right)= & (1-\hat{\delta}) u_{i}\left(\pi^{0}(f)\right) \\
& +(1-\hat{\delta}) \mathrm{E}\left(\delta_{0}^{1} u_{i}\left(\pi^{1}(f)\left(d^{1}\right)\right) \mid \mathcal{F}_{0}\right) \\
& +(1-\hat{\delta}) \mathrm{E}\left(\mathrm{E}\left(\delta_{0}^{2} u_{i}\left(\pi^{2}(f)\left(d^{2}\right)\right) \mid \mathcal{F}_{1}\right) \mid \mathcal{F}_{0}\right) \\
& +(1-\hat{\delta}) \mathrm{E}\left(\mathrm{E}\left(\mathrm{E}\left(\delta_{0}^{3} u_{i}\left(\pi^{3}(f)\left(d^{3}\right)\right) \mid \mathcal{F}_{2}\right) \mid \mathcal{F}_{1}\right) \mid \mathcal{F}_{0}\right)+\ldots
\end{aligned}
$$

Because that $\left\{\mathcal{F}_{s}\right\}_{s=0,1,2, \ldots}$ is a filtration, the above term reduces to

$$
\begin{aligned}
U_{i}\left(f,\left\{\mathbf{d}_{t}\right\}_{t}\right)= & (1-\hat{\delta}) u_{i}\left(\pi^{0}(f)\right) \\
& +(1-\hat{\delta}) \mathrm{E}\left(\delta_{0}^{1} u_{i}\left(\pi^{1}(f)\left(d^{1}\right)\right) \mid \mathcal{F}_{0}\right) \\
& +(1-\hat{\delta}) \mathrm{E}\left(\delta_{0}^{2} u_{i}\left(\pi^{2}(f)\left(d^{2}\right)\right) \mid \mathcal{F}_{0}\right) \\
& +(1-\hat{\delta}) \mathrm{E}\left(\delta_{0}^{3} u_{i}\left(\pi^{3}(f)\left(d^{3}\right)\right) \mid \mathcal{F}_{0}\right)+\ldots,
\end{aligned}
$$

i.e.

$$
U_{i}\left(f,\left\{\mathbf{d}_{t}\right\}_{t}\right)=(1-\hat{\delta}) \sum_{k=0}^{\infty} \mathrm{E}\left(\delta_{0}^{k} u_{i}\left(\pi^{k}(f)\left(d^{k}\right)\right) \mid \mathcal{F}_{0}\right)
$$


where $\pi^{0}(f)\left(d^{0}\right)=\pi(f(e))$, and recall that $\mathrm{E}\left(\delta_{t}^{t} \mid \mathcal{F}_{s}\right)=1$ for all $s \leq t$. Following a similar method, we can also define the continuation utility of player $i$ as follows: Given $t \in \mathbb{N}$ and $d^{t} \in \mathbb{R}^{t}$ for $\tau \geq t$

$$
V_{i}^{\tau, d^{t}}\left(f,\left\{\mathbf{d}_{t}\right\}_{t}\right)=(1-\hat{\delta}) \sum_{k=\tau}^{\infty} \mathrm{E}\left(\delta_{\tau}^{k} u_{i}\left(\pi^{k}(f)\left(d^{k}\right)\right) \mid \mathcal{F}_{t}\right) .
$$

We use the convention that $V_{i}^{0, d^{0}}\left(f,\left\{\mathbf{d}_{t}\right\}_{t}\right)=U_{i}\left(f,\left\{\mathbf{d}_{t}\right\}_{t}\right)$.

When attention is restricted to $\bar{G}(\hat{\delta})$, i.e. the repeated game with constant discounting, the payoffs are defined as follows: For any strategy $\bar{f}$ of the repeated game $\bar{G}(\hat{\delta})$, the payoff of player $i$ is given by $\bar{U}_{i}(\bar{f}, \hat{\delta})=(1-\hat{\delta}) \sum_{t=0}^{\infty} \hat{\delta}^{t} u_{i}\left(\bar{\pi}^{t}(\bar{f})\right)$, where $\bar{\pi}(\bar{f}) \in A^{\infty}$ is the outcome path of $\bar{G}(\hat{\delta})$ induced by $\bar{f}$. For any $\bar{\pi} \in A^{\infty}, t \in \mathbb{N}_{0}$, and $i \in N$, let $\bar{V}_{i}^{t}(\bar{\pi}, \hat{\delta})=(1-\hat{\delta}) \sum_{r=t}^{\infty} \hat{\delta}^{r-t} u_{i}\left(\bar{\pi}^{r}\right)$ be the continuation payoff of player $i$ at date $t$ if the outcome path $\bar{\pi}$ is played.

\section{Subgame Perfect Equilibria}

A strategy vector $f \in F$ is a Nash equilibrium of $G\left(\left\{\mathbf{d}_{t}\right\}_{t}\right)$ if for all $i \in N, U_{i}\left(f,\left\{\mathbf{d}_{t}\right\}_{t}\right) \geq$ $U_{i}\left(\left(\hat{f}_{i}, f_{-i}\right),\left\{\mathbf{d}_{t}\right\}_{t}\right)$ for all $\hat{f}_{i} \in F_{i}$. A strategy vector $f \in F$ is a subgame perfect equilibrium of the supergame $G\left(\left\{\mathbf{d}_{t}\right\}_{t}\right)$ if every $f^{\prime} \in F(f)$ is a Nash equilibrium. We denote the set of subgame perfect equilibrium strategies of $G\left(\left\{\mathbf{d}_{t}\right\}_{t}\right)$ by $\operatorname{SPE}\left(G\left(\left\{\mathbf{d}_{t}\right\}_{t}\right)\right)$. Let $\mathcal{V}\left(\left\{\mathbf{d}_{t}\right\}_{t}\right)$ be the subgame perfect equilibrium payoffs of $G\left(\left\{\mathbf{d}_{t}\right\}_{t}\right)$. We will abuse notation and will let $\mathcal{V}\left(\left\{\mathbf{d}_{t}\right\}_{t}\right)$ denoted by $\mathcal{V}(\hat{\delta})$ where $\hat{\delta}=\mathbf{d}_{0}$. Moreover, $\mathcal{V}\left(\left\{\mathbf{d}_{t}\right\}_{t}, \tau\right)$ are the subgame perfect equilibrium continuation payoffs (in period $\tau$ terms), when $d^{\tau}$ is realized. In fact, abusing notation we let $\mathcal{V}\left(\left\{\mathbf{d}_{t}\right\}_{t}, \tau\right)=\mathcal{V}\left(\delta_{\tau}^{\tau+1}\right)$.

Moreover, when attention is restricted to the repeated game with constant discounting, $\bar{G}(\hat{\delta})$, subgame perfection can easily be defined by excluding stochastic parts of the above definitions. We denote the set of subgame perfect strategies in $\bar{G}(\hat{\delta})$ by $\operatorname{SPE}(\bar{G}(\hat{\delta}))$. Let $\overline{\mathcal{V}}(\hat{\delta})$ be the set of subgame perfect equilibrium payoffs in the repeated game with constant discount factor $\hat{\delta}$. 
Letting $\mathbf{d}_{0}=\hat{\delta}$ below we will show that for every $t$ and $d^{t}, \mathcal{V}\left(\delta_{t}^{t+1}\right)$ is compact, hence, obtain the following characterization analogous to Abreu (1988): A strategy $f$ is subgame perfect if and only if for all $i \in N$ and for all $t \in \mathbb{N}_{0}$ and for all $d^{t} \in \mathbb{R}^{t}$, we have

$$
V_{i}^{t, d^{t}}\left(f,\left\{\mathbf{d}_{t}\right\}_{t}\right) \geq(1-\hat{\delta}) \max _{a_{i} \in A_{i}} u_{i}\left(a_{i}, \pi_{-i}^{t}(f)\left(d^{t}\right)\right)+\delta_{t}^{t+1} \mathrm{E}\left(v_{i}\left(d^{t+1}\right) \mid \mathcal{F}_{t}\right),
$$

where $\delta_{t}^{t+1}=\mathbf{d}_{t}^{t+1}$ (i.e. given $d^{t}$, the realization of $\mathbf{d}_{t}^{t+1}$ is equal to $\delta_{t}^{t+1}$ ), and for every $i \in N$

$$
v_{i}\left(d^{t+1}\right)=\min \left\{u_{i}: u_{i} \in \mathcal{V}\left(\delta_{t+1}^{t+2}\right)\right\}
$$

Before the justification of these, we wish to describe the resulting construction briefly. Notice that, when player $i$ decides whether or not to follow the equilibrium behavior in period $t$ given the history of process, $d^{t}$, it must be that: The player $i$ 's expected continuation payoff associated with the equilibrium behavior must be as high as player $i$ deviating singly and optimally today, and being punished tomorrow. An important issue to notice is that, tomorrow players will observe $d_{t+1}$ (thus, $\delta_{t+1}^{t+2}$ ) before deciding on their actions. Thus, players will be punishing player $i$, the deviator, with the most severe and credible punishment with the information they have in period $t+1$. Thus, the punishment payoff to player $i$ with the information that players have in period $t+1$, i.e. $d^{t+1}$, is $v_{i}\left(d^{t+1}\right)$. Player $i$ forecasts these in period $t$, and hence, forms an expectation regarding his punishment payoff (starting from period $t+1$ onwards) with the information that he has in period $t$, namely $d^{t}$.

In order to show that for every $t$ and $d^{t}, \mathcal{V}\left(\delta_{t}^{t+1}\right)$ is compact, we will be employing the construction of Abreu, Pearce, and Stachetti (1990), and it is important to point out that their assumptions, $1-5$ are all satisfied in our framework: A2, A3, and A4 are trivially satisfied as the period payoffs are deterministic, and we also impose A1 and A5.

Following their construction, given $d^{t}$ for any $W \subset \mathbb{R}^{N}$ and the resulting level of $\delta_{t}^{t+1} \in(0,1)$, let $g\left(\delta_{t}^{t+1}\right)$ be the expected discounted continuation utility vector 
(not including today's payoff levels and using the normalization via $\hat{\delta} \in(0,1)$ ) for an arbitrary strategy profile. Furthermore, for that given level of $\delta_{t}^{t+1}$, consider the pair, $\left(g\left(\delta_{t}^{t+1}\right), a\right)$ and define $\mathrm{E}\left(g\left(\delta_{t}^{t+1}\right), a\right)=\delta_{t}^{t+1}\left((1-\hat{\delta}) u(a)+g\left(\delta_{t+1}^{t+2}\right)\right)$. A pair $\left(g\left(\delta_{t}^{t+1}\right), a\right)$ is called admissible with respect to $W$, whenever $\mathrm{E}\left(g_{i}\left(\delta_{t}^{t+1}\right), a\right) \geq$ $\mathrm{E}\left(g_{i}\left(\delta_{t}^{t+1}\right),\left(\gamma_{i}, a_{-i}\right)\right)$ for all $\gamma_{i} \in A_{i}$ and for all $i \in N$. Moreover, for each set $W$, define $B^{d^{t}}(W)$ as follows $B^{d^{t}}(W)=\left\{\mathrm{E}\left(g\left(\delta_{t}^{t+1}\right), a\right) \mid\left(g\left(\delta_{t}^{t+1}\right), a\right)\right.$ is admissible w.r.t $\left.W\right\}$. Any set that satisfies $W \subset B^{d^{t}}(W)$ is called self-generating at $d^{t}$. At this point it is useful to recall that $\mathcal{V}\left(\delta_{t}^{t+1}\right)=\left\{V^{t, d^{t}}\left(f,\left\{\mathbf{d}_{t}\right\}_{t}\right) \mid f \in S P E\left(G\left(\left\{\mathbf{d}_{t}\right\}_{t}\right)\right)\right\}$.

Notice that

$$
g\left(\delta_{t}^{t+1}\right)=(1-\hat{\delta}) \sum_{k=t+1}^{\infty} \mathrm{E}\left(\delta_{t}^{k} u\left(\pi^{k}(f)\left(d^{k}\right)\right) \mid \mathcal{F}_{t}\right)
$$

for some strategy profile $f$. Furthermore since, $\delta_{t}^{t+1}$ is actually realized before the actions are taken and the multiplicative nature of our discount factor, the above equation becomes

$$
g\left(\delta_{t}^{t+1}\right)=(1-\hat{\delta}) \delta_{t}^{t+1}\left[u\left(\pi^{t+1}(f)\left(d^{t}\right)\right)+\sum_{k=t+2}^{\infty} \mathrm{E}\left(\delta_{t}^{k} u\left(\pi^{k}(f)\left(d^{k}\right)\right) \mid \mathcal{F}_{t}\right)\right]
$$

which is equal to

$$
g\left(\delta_{t}^{t+1}\right)=\delta_{t}^{t+1}\left[(1-\hat{\delta}) u\left(\pi^{t+1}(f)\left(d^{t}\right)\right)+g\left(\delta_{t+1}^{t+2}\right)\right]
$$

Now, it is easy to see that $\mathcal{V}\left(\delta_{t}^{t+1}\right)$ is self-generating, as the pair, $\left(g\left(\delta_{t}^{t+1}\right), \pi^{t+1}(f)\left(d^{t}\right)\right)$ is admissible with respect to $\mathcal{V}\left(\delta_{t}^{t+1}\right)$, whenever $f$ is a subgame perfect equilibrium strategy profile with

$$
V^{t, d^{t}}\left(f,\left\{\mathbf{d}_{t}\right\}_{t}\right)=(1-\hat{\delta}) u\left(a_{t}\right)+g\left(\delta_{t}^{t+1}\right)
$$

where $a_{t}=\pi^{t}(f)\left(d^{t-1}\right)$. The two further points to notice is that, due to Lemma 1 of Abreu, Pearce, and Stachetti (1990), $B^{d^{t}}(W)$ is compact whenever $W$ is compact, and the operator $B^{d^{t}}$ is monotone. Furthermore, since $\mathcal{V}\left(\delta_{t}^{t+1}\right)$ is bounded (by $\left.\left(1 / 1-\delta_{t}^{t+1}\right) \mathcal{U}\right)$, closure of $\mathcal{V}\left(\delta_{t}^{t+1}\right)$, denoted by $\operatorname{cl}\left(\mathcal{V}\left(\delta_{t}^{t+1}\right)\right)$ is compact. Hence, 
$B^{d^{t}}\left(\operatorname{cl}\left(\mathcal{V}\left(\delta_{t}^{t+1}\right)\right)\right)$ is compact, and due to $\operatorname{cl}\left(\mathcal{V}\left(\delta_{t}^{t+1}\right)\right) \subset B^{d^{t}}\left(\operatorname{cl}\left(\mathcal{V}\left(\delta_{t}^{t+1}\right)\right)\right)$ and selfgeneration $\operatorname{cl}\left(\mathcal{V}\left(\delta_{t}^{t+1}\right)\right) \subset \mathcal{V}\left(\delta_{t}^{t+1}\right)$. Thus, by Theorem 2 of Abreu, Pearce, and Stachetti $(1990), B^{d^{t}}\left(\mathcal{V}\left(\delta_{t}^{t+1}\right)\right)=\mathcal{V}\left(\delta_{t}^{t+1}\right)$, thus $\mathcal{V}\left(\delta_{t}^{t+1}\right)$ is compact.

\section{Inevitability of Nash behavior}

In this section, we wish to present the main result of this study:

Theorem 1 Suppose Assumptions 1, 2, 3 hold. Then, for every $K \in \mathbb{N}$, for every $\hat{\delta} \in(0,1)$, for every stochastic discounting process $\left\{\mathbf{d}_{t}\right\}_{t}$ with $\mathbf{d}_{0}=\hat{\delta}$, and for every subgame perfect strategy profile $f$ of the repeated game with stochastic discounting; there exists $T$ which is almost surely in $\mathbb{N}_{0}$, and the probability of $\pi^{\tau}(f)$ being a Nash equilibrium action profile of the stage game conditional on the information available at $s$, equals 1, for all $s=T, \ldots, T+K$ and for all $\tau=s, \ldots, T+K$.

The above theorem establishes that when Assumptions 1, 22 and 3 hold, then arbitrary long (yet, finite) consecutive repetitions of the period Nash action profile must almost surely happen in a finite time window no matter which subgame perfect equilibrium strategy is considered and no matter how high the initial discount factor is. That is, any equilibrium strategy almost surely entails arbitrarily long consecutive observations of the period Nash action profile.

Showing this result involves 2 steps: The first displays that every subgame perfect strategy must involve the prescription of the Nash behavior whenever the current discount factor is sufficiently small. The second displays that for any given level of the initial discount factor and any given natural number $K$, the stochastic process governing the one-shot discount factors possesses a stopping time, after which the return to some sufficiently high level of one-shot discount rates within a $K$-period time window, has zero probability with the evaluation being made in any period within that time window.

The Proof of Theorem 1. The result follows from Lemmas 2 and 3 . 
Lemma 2 Suppose Assumptions 1, 2, 3 hold, and let $\hat{\delta} \in(0,1)$. Then, for every subgame perfect strategy profile $f$ of $G\left(\left\{\mathbf{d}_{t}\right\}\right)$ with $\mathbf{d}_{0}=\hat{\delta}$, there exists $\underline{\delta} \in(0,1)$ such that for all $\delta_{t}^{t+1} \leq \underline{\delta}, t \in \mathbb{N}_{0}$, it must be such that $f\left(d^{t}, a^{t}\right) \in A$ is a Nash equilibrium of $G$.

Proof. Without loss of generality, assume that the subgame perfect strategy $f$ is such that $\left(\max _{a_{i} \in A_{i}} u_{i}\left(a_{i}, \pi_{-i}^{t}(f)\left(d^{t}\right)\right)-u_{i}\left(\pi^{t}(f)\left(d^{t}\right)\right)\right)>0$ for some $t \in \mathbb{N}_{0}$ and for some $d^{t}$ and for some $i \in N$. Because otherwise, the strategy is resulting in a repetition of period Nash behavior. Then, by equation 3 , for any such subgame perfect strategy $f$ and $i$ and $t$ and $d^{t}$

$$
\delta_{t}^{t+1}\left(V_{i}^{t+1, d^{t}}-\mathrm{E}\left(v_{i}\left(d^{t+1}\right) \mid \mathcal{F}_{t}\right)\right) \geq(1-\hat{\delta})\left(\max _{a_{i} \in A_{i}} u_{i}\left(a_{i}, \pi_{-i}^{t}(f)\left(d^{t}\right)\right)-u_{i}\left(\pi^{t}(f)\left(d^{t}\right)\right)\right) .
$$

Both the left and the right hand sides of this inequality are strictly positive. Yet, when the prescribed action is not a Nash equilibrium of $G$, then the left hand side converges to 0 when $\delta_{t}^{t+1}$ tends to 0 , but the right hand side is constant.

Lemma 3 Suppose Assumptions 1, 2, 3 hold. Then, for every $\underline{\delta} \in(0,1)$, for every $K \in \mathbb{N}$, for every $\hat{\delta} \in(0,1)$, and for every stochastic discounting process $\left\{\mathbf{d}_{t}\right\}_{t}$ with $\hat{\delta}=\mathbf{d}_{\mathbf{0}}$; there exists $T$ which is almost surely in $\mathbb{N}_{0}$ and $\operatorname{Pr}\left[\mathbf{d}_{\tau}^{\tau+1}<\underline{\delta} \mid \mathcal{F}_{s}\right]=1$, for every $s=T, \ldots, T+K$ and $\tau=s, \ldots, T+K$.

Proof. Let $\underline{\delta} \in(0,1)$ and $K \in \mathbb{N}$ and $\hat{\delta} \in(0,1)$ with $\hat{\delta}=\mathbf{d}_{\mathbf{0}}$. Let $\omega_{0} \in\{\omega \in$ $\left.\Omega^{E}: \omega<\underline{\delta}\right\} \neq \emptyset$ due to part (4) and (5) of Assumption 2. Consider $\omega_{1} \in \Omega^{E}$ with $\omega_{0} \geq \max R\left(\omega_{1}\right)$, and such an $\omega_{1}$ exists due to part (4), (5) and (6) of Assumption 2. 9 Now, define $\omega_{2} \in \Omega^{E}$ that satisfies $\omega_{1} \geq \max R\left(\omega_{2}\right)$. Inductively, for a given $\omega_{K-1} \in \Omega^{E}$ define $\omega_{K} \in \Omega^{E}$ likewise. Again notice that due to Assumption 2 such an $\omega_{K}$ exists.

\footnotetext{
${ }^{9}$ Recall that for any given state $\omega \in \Omega \subseteq(0,1)$, the set of states $\omega^{\prime} \in \Omega$ that are reachable from $\omega$ in a single period and satisfying $\omega<\omega^{\prime}$, is denoted by $R(\omega)$.
} 
Following Karlin and Taylor (1975), define the following event

$$
\zeta \equiv \min \left\{\tau \in \mathbb{N}_{0}: \delta_{\tau}^{\tau+1} \leq \omega_{K}\right\}
$$

Then by construction, it must be that $\operatorname{Pr}\left[\mathbf{d}_{s+k} \geq \underline{\delta} \mid \mathcal{F}_{s}\right]=0$, for all $s=\zeta, \ldots, \zeta+K$ and $k=0, \ldots, K-s$. Finally, due to the ergodicity of $\omega_{K}, \zeta$ is a stopping time, that is it will almost surely happen in a finite time period, i.e. $\operatorname{Pr}[\zeta<\infty]=1$. Hence, $\zeta$ is almost surely in $\mathbb{N}_{0}$ with $\operatorname{Pr}\left[\mathbf{d}_{s+k}<\underline{\delta} \mid \mathcal{F}_{s}\right]=1$ for all $s=\zeta, \ldots, \zeta+K$ and $k=0, \ldots, K-s .10$

\section{The Subgame Perfect Folk Theorem}

In this section we prove the following subgame perfect Folk Theorem for repeated games with stochastic discounting.

Theorem 2 Suppose Assumptions 1, 2, 3 hold, and either $\operatorname{dim}(\mathcal{U})=n$ or $n=2$ and $\mathcal{U}^{0} \neq \emptyset$. Then, for all $\varepsilon>0$, there exists $\underline{\delta} \in(0,1)$ such that for all $u \in \mathcal{U}^{0}$ and for all stochastic discounting processes $\left\{\mathbf{d}_{t}\right\}_{t \in \mathbb{N}_{0}}$ with $\hat{\delta}=\mathbf{d}_{0} \geq \underline{\delta}$, there exists a subgame perfect strategy $f$ of $G\left(\left\{\mathbf{d}_{t}\right\}_{t}\right)$ such that $\left\|U\left(f,\left\{\mathbf{d}_{\mathbf{t}}\right\}_{t}\right)-u\right\|<\varepsilon$.

In order to establish this result, an analogy between repeated games with stochastic discounting and those with constant discounting is constructed as follows: Given any repeated game with stochastic discounting, we consider the repeated game with a constant discount factor that equals the initial level of the stochastic discounting process. Particularly, in the repeated game with constant discounting we concentrate on strictly enforceable strategies, those to which players strictly prefer to conform, in each date and state including equilibrium and punishment phases. It is useful to remind the reader that due to the monotonicity result of Abreu, Pearce, and Stachetti (1990), Theorem 6, such strategies are strictly enforceable for higher discount factors as well.

\footnotetext{
${ }^{10}$ Indeed, this also implies that $\zeta$ is almost surely in $\mathbb{N}_{0}$ with $\mathrm{E}_{\tau}\left(\mathbf{d}_{\tau}\right)<\underline{\delta}$, for every $\tau=\zeta, \ldots, \zeta+K$.
} 
Formulating an extension of such a strategy and requiring it to be subgame perfect in the repeated game with stochastic discounting, turns out to be an arduous, yet feasible (as proven in Lemma 4), endeavor whenever the initial level of the stochastic discounting is sufficiently high.

To see the difficulties involved, consider a repeated prisoners' dilemma, with actions $A_{i}=\{c, d\}$ and $u_{1}(d, c)=u_{2}(c, d)>u_{i}(c, c)>\frac{1}{2}\left(u_{1}(c, d)+u_{2}(c, d)\right)>u_{i}(d, d)>$ $u_{1}(c, d)=u_{2}(d, c), i=1,2$. Clearly, there exists $\underline{\delta} \in(0,1)$ such that the cooperative payoff (hence, path given by $((c, c) ; \infty)$ ) is sustained with a strictly enforceable strategy profile for all $\delta>\underline{\delta}$. Now, consider any stochastic discounting process satisfying our restrictions and possessing a sufficiently high initial level, and any strategy such that its utility (evaluated at the beginning of the game) is arbitrarily close to $\left(u_{i}(c, c)\right)_{i=1,2}$. Due to Lemma 2, for any realizations of the one-shot discount factor that is strictly below $\underline{\delta}$, any such strategy must dictate the play of $(d, d)$, if it were to be subgame perfect. Thus, any subgame perfect strategy in the repeated game with stochastic discounting sustaining the cooperative payoff must be contingent on the realizations of the one-shot discount factors. A simple formulation is one where this contingency is represented by a date and state independent threshold, $\delta_{*} \geq \underline{\delta}$, so that: the play continues on the cooperative path as long as every past realization of the one-shot discount factors is above $\delta_{*}$; and otherwise, the play switches to the defection phase. Then, the verification of subgame perfection in the repeated game with stochastic discounting calls for checking every subgame, in particular, those with the current one-shot discount factor arbitrarily close, yet, strictly exceeding $\delta_{*}$. In such a subgame where additionally there have not been any single player deviations in the past and all past one-shot discount factors have been above $\delta_{*}$, this strategy should call for the play of $(c, c)$. However, it is not subgame perfect whenever the following holds: The stochastic discounting process is one where the probability of the next period's one-shot discount factor being strictly less than $\delta_{*}$, is high enough such that any one of the players finds it profitable to deviate in the current period. 
Therefore, given a strictly enforceable strategy in the repeated game with constant discounting, the extended strategy we employ is contingent on the stochastic discounting process in the following manner: It will prescribe the play to continue on the paths dictated by its counterpart in the repeated game with constant discounting, whenever each of the past realizations of the one-shot discount factors exceeds a date and state specific threshold. Otherwise, our strategy will recommend the play to consist of the repetitions of a Nash action profile of the stage game thereafter. The initial level of the stochastic discounting process can be chosen sufficiently high so that we can construct date and state specific thresholds such that, given a date and state, the probability (evaluated in that date and state) of the one-shot discount factor in the next period falling below its associated threshold, is sufficiently low. This and strict enforceability, in turn, imply that the relevant incentive conditions hold for any date and state. Meanwhile, choosing the initial level of the stochastic discounting process to be sufficiently high, also results in the utility (evaluated at the beginning of the game) of this strategy profile in the repeated game with stochastic discounting to be arbitrarily close to the utility of its counterpart in the game with the constant discount factor given by that initial level.

Finally, our result is obtained by combining the above construction and the observation that when restricted to pure actions, the strategy profile in the proof of the subgame perfect Folk Theorem of Fudenberg and Maskin (1991) is, in fact, strictly enforceable.

The rest of this section presents the details about the proof of Theorem 2 .

Suppose Assumptions 1, 2, and 3 hold. Then, for any $\hat{\delta} \in(0,1)$, consider the repeated game with stochastic discounting $G\left(\left\{\mathbf{d}_{\mathbf{t}}\right\}_{t}\right)$ with $\mathbf{d}_{\mathbf{0}}=\hat{\delta}$; and, the repeated game with constant discounting $\bar{G}(\hat{\delta})$. For any $k$-stage history $h_{k}=$ $\left(\left(a_{0}, d_{1}\right), \ldots,\left(a_{k-1}, d_{k}\right)\right)$ of $G\left(\left\{\mathbf{d}_{\mathbf{t}}\right\}_{t}\right)$ where for all $0 \leq t \leq k-1, a_{t} \in A$, and for all $1 \leq t \leq k, d_{t}$ is realization of $\mathbf{d}_{t}$, define its deterministic counterpart, a $k$-stage history, in $\bar{G}(\hat{\delta})$ by $\bar{h}_{k}=\left(a_{0}, \ldots, a_{k-1}\right)$. 
Following Abreu (1988), it is well known that one may restrict attention to simple strategies in the analysis of subgame perfection in repeated games with constant discounting: $\bar{f}$ in $\bar{G}(\delta), \delta \in(0,1)$ is a simple strategy profile represented by $n+1$ paths $\left(\bar{\pi}^{(0)}, \bar{\pi}^{(1)}, \ldots, \bar{\pi}^{(n)}\right)$ if $\bar{f}$ specifies: (i) play $\bar{\pi}^{(0)}$ until some player deviates singly from $\bar{\pi}^{(0)}$; (ii) for any $j \in N$, play $\bar{\pi}^{(j)}$ if the $j$ th player deviates singly from $\bar{\pi}^{(i)}$, $i=0,1, \ldots, n$, where $\bar{\pi}^{(i)}$ is the ongoing previously specified path; (iii) continue with the ongoing specified path $\bar{\pi}^{(i)}, i=0,1, \ldots, n$, if no deviations occur or if two or more players deviate simultaneously. These strategies are simple because the play of the game is always in only $(n+1)$ states, namely, in state $j \in\{0, \ldots, n\}$ where $\bar{\pi}^{(j), t}$ is played, for some $t \in \mathbb{N}_{0}$. In this case, we say that the play is in phase $t$ of state $j$. A profile $\left(\bar{\pi}^{(0)}, \bar{\pi}^{(1)}, \ldots, \bar{\pi}^{(n)}\right)$ of $n+1$ outcome paths is subgame perfect if the simple strategy represented by it is a subgame perfect equilibrium. Moreover, following Barlo, Carmona, and Sabourian (2009), we say that a simple strategy $\bar{f}$ in $\bar{G}(\delta), \delta \in[0,1)$, is weakly enforceable if for all $i \in N$ and for all $j \in\{0,1, . ., n\}$ and for all $t \in \mathbb{N}_{0}$

$$
\bar{V}_{i}^{t}\left(\bar{\pi}^{(j)}, \delta\right) \geq(1-\delta) \max _{a_{i} \in A_{i}} u_{i}\left(a_{i}, \bar{\pi}_{-i}^{(j), t}\right)+\delta \bar{V}_{i}^{t+1}\left(\bar{\pi}^{(i)}, \delta\right),
$$

where $\left(\bar{\pi}^{(0)}, \bar{\pi}^{(1)}, \ldots, \bar{\pi}^{(n)}\right)$ is the simple strategy associated with $\bar{f}$. Due to Abreu (1988), we know that a simple strategy $\bar{f} \in S P E(\bar{G}(\delta))$ if and only if $\bar{f}$ in $\bar{G}(\delta)$ is weakly enforceable. Moreover, we say that a simple strategy $\bar{f}$ in $\bar{G}(\delta)$ with associated outcome paths $\left(\bar{\pi}^{(0)}, \bar{\pi}^{(1)} \ldots, \bar{\pi}^{(n)}\right)$ is strictly enforceable if for all $i \in N$ and for all $j \in\{0,1, . ., n\}$ and for all $t \in \mathbb{N}_{0}$

$$
\inf _{i, j, t}\left(\bar{V}_{i}^{t}\left(\bar{\pi}^{(j)}, \delta\right)-\left((1-\delta) \max _{a_{i} \in A_{i}} u_{i}\left(a_{i}, \bar{\pi}_{-i}^{(j), t}\right)+\delta \bar{V}_{i}^{t+1}\left(\bar{\pi}^{(i)}, \delta\right)\right)\right)>0
$$

Let $\bar{f}$ be a strictly enforceable simple strategy in $\bar{G}(\delta), \delta \in(0,1)$, and the profile of outcome paths associated be $\left(\bar{\pi}^{(0)}, \bar{\pi}^{(1)}, \ldots, \bar{\pi}^{(n)}\right)$. Now, let us formulate an analogy between $G\left(\left\{\mathbf{d}_{\mathbf{t}}\right\}_{t}\right)$ with $\mathbf{d}_{0}=\hat{\delta}$ and $\bar{G}(\hat{\delta})$ for $\hat{\delta} \geq \delta$.

Lemma 4 Suppose that Assumptions 1, 2, 3 hold, and $\bar{f}$ of $\bar{G}(\delta)$, where $\delta \in(0,1)$ and the associated outcome paths are $\left(\bar{\pi}^{(0)}, \bar{\pi}^{(1)}, \ldots, \bar{\pi}^{(n)}\right)$, is a strictly enforceable simple 
strategy. Then, for all $\eta>0$ there exists $\delta^{*} \in(\delta, 1)$ such that for all $\hat{\delta}>\delta^{*}$ there is $f$ in $G\left(\left\{\mathbf{d}_{t}\right\}_{t}\right)$ with $\mathbf{d}_{0}=\hat{\delta}$, such that $f$ is subgame perfect in $G\left(\left\{\mathbf{d}_{t}\right\}_{t}\right)$ and

$$
\left\|U\left(f,\left\{\mathbf{d}_{t}\right\}_{t}\right)-\bar{U}(\bar{f}, \hat{\delta})\right\|<\eta \text {. }
$$

Proof. Let $\nu^{*}$ be defined by

$$
\nu^{*} \equiv \inf _{i, j, t}\left(\bar{V}_{i}^{t}\left(\bar{\pi}^{(j)}, \delta\right)-\left((1-\delta) \max _{a_{i} \in A_{i}} u_{i}\left(a_{i}, \bar{\pi}_{-i}^{(j), t}\right)+\delta \bar{V}_{i}^{t+1}\left(\bar{\pi}^{(i)}, \delta\right)\right)\right)>0,
$$

and consider $\nu>0$ with $\nu<\min \left\{\nu^{*}, \eta\right\}$. Then, there exists $\delta_{\nu}>\delta$ and $p_{\nu} \in(0,1)$ sufficiently close to 0 such that the following conditions hold: For all $i \in N, j \in$ $N \cup\{0\}, t \in \mathbb{N}_{0}$

$$
\begin{aligned}
& \left\|\delta_{\nu} \bar{V}_{i}^{t+1}\left(\bar{\pi}^{(j)}, \delta_{\nu}\right)-\left(\delta_{\nu}\left(\left(1-p_{\nu}\right) \bar{V}_{i}^{t+1}\left(\bar{\pi}^{(j)}, \delta_{\nu}\right)+p_{\nu} \bar{V}_{i}^{t+1}\left(a^{*}, \delta_{\nu}\right)\right)\right)\right\|<\frac{\nu}{6} \\
& \inf _{i, j, t}\left[\left(1-\delta_{\nu}\right) u_{i}\left(\bar{\pi}^{(j), t}\right)+\delta_{\nu}\left(\left(1-p_{\nu}\right) \bar{V}_{i}^{t+1}\left(\bar{\pi}^{(j)}, \delta_{\nu}\right)+p_{\nu} \bar{V}_{i}^{t+1}\left(a^{*}, \delta_{\nu}\right)\right)\right. \\
& \quad-\left(\left(1-\delta_{\nu}\right) \max _{a_{i} \in A_{i}} u_{i}\left(a_{i}, \bar{\pi}_{-i}^{(j), t}\right)+\delta_{\nu}\left(\left(1-p_{\nu}\right) \bar{V}_{i}^{t+1}\left(\bar{\pi}^{(i)}, \delta_{\nu}\right)+p_{\nu} \bar{V}_{i}^{t+1}\left(a^{*}, \delta_{\nu}\right)\right)\right]>\nu \\
& \left\|\sum_{t=0}^{\infty} \mathrm{E}_{0}\left(\delta_{0}^{t}\right)\left(u_{i}\left(\bar{\pi}^{t}(\bar{f})\right)\right)-\sum_{t=0}^{\infty}\left(\delta_{\nu}\right)^{t}\left(u_{i}\left(\bar{\pi}^{t}(\bar{f})\right)\right)\right\|<\frac{\nu}{6}, \\
& \left\|\sum_{t=0}^{\infty} \mathrm{E}_{0}\left(\delta_{0}^{t}\right)\left(u_{i}\left(a^{*}\right)\right)-\sum_{t=0}^{\infty}\left(\delta_{\nu}\right)^{t}\left(u_{i}\left(a^{*}\right)\right)\right\|<\frac{\nu}{6},
\end{aligned}
$$

Condition 4 holds trivially. On the other hand, condition 5 holds because $\bar{f}$ is strict enforceable at $\delta$, and the monotonicity result, Theorem 6 of Abreu, Pearce, and Stachetti (1990), implies that $\bar{f} \in S P E\left(\bar{G}\left(\delta^{\prime}\right)\right)$ for any $\delta^{\prime} \geq \delta$. Indeed, it can easily be verified (by using the same techniques of the proof of this result) that $\bar{f}$ is also strictly enforceable at $\delta^{\prime} \geq \delta$. Furthermore, since $p_{\nu}$ can be selected arbitrarily close to 0 , the associated slack (the left hand side of condition 5 , which converges to $\nu^{*}$ when $p_{\nu}$ tends to 0 ) can be chosen to strictly exceed $\nu$. Moreover, conditions 6 and 7 are due to the following: Observe that for any process satisfying Assumption 2 with $\mathbf{d}_{0}=\delta_{\nu}$, the fourth part of Lemma 1 and the Sandwich Lemma directly imply that as $\delta_{\nu}$ tends to $1, \mathrm{E}\left(\delta_{t}^{\tau} \mid \mathcal{F}_{0}\right)$ tends to $\left(\delta_{\nu}\right)^{\tau-t}$ for all $\tau, t \in \mathbb{N}_{0}$ with $\tau \geq t$. It is important 
to point out that because $p_{\nu}$ can be selected arbitrarily small, all these conditions, 4 - 7, keep holding when they are evaluated at $p_{\nu}$ and $\hat{\delta}>\delta_{\nu}$ and $\mathbf{d}_{0}=\hat{\delta}$.

Furthermore, observe that since $\left\{\mathbf{d}_{t}\right\}_{t}$ is a non-negative bounded martingale, $\left\{\mathbf{e}_{t}\right\}_{t}$ defined by $\mathbf{e}_{t} \equiv\left(1-\mathbf{d}_{t}\right)$ for all $t \in \mathbb{N}_{0}$ is also a non-negative, bounded martingale. Using Doob's Maximal Inequality (we refer the reader to Doob (1984)) 11 for this martingale we obtain for any $t<T$ and $\bar{\delta} \in(0,1)$

$$
\begin{aligned}
& (1-\bar{\delta}) \operatorname{Pr}\left[\sup _{t \leq s \leq T}\left(1-\delta_{s}^{s+1}\right) \geq(1-\bar{\delta}) \mid \mathcal{F}_{t}\right] \leq \mathrm{E}\left(\left(1-\delta_{T}^{T+1}\right) \mid \mathcal{F}_{t}\right) \\
& (1-\bar{\delta}) \operatorname{Pr}\left[\inf _{t \leq s \leq T} \delta_{s}^{s+1} \leq \bar{\delta} \mid \mathcal{F}_{t}\right] \leq \mathrm{E}\left(\left(1-\delta_{T}^{T+1}\right) \mid \mathcal{F}_{t}\right) \\
& \operatorname{Pr}\left[\inf _{t \leq s \leq T} \delta_{s}^{s+1} \leq \bar{\delta} \mid \mathcal{F}_{t}\right] \leq \frac{\mathrm{E}\left(\left(1-\delta_{T}^{T+1}\right) \mid \mathcal{F}_{t}\right)}{(1-\bar{\delta})} .
\end{aligned}
$$

Moreover, since $\left\{\mathbf{d}_{t}\right\}_{t}$ is a martingale the right hand side of the above condition is constant for all $T \in \mathbb{N}_{0}$ and $t<T$, i.e.

$$
\operatorname{Pr}\left[\inf _{t \leq s \leq T} \delta_{s}^{s+1} \leq \bar{\delta} \mid \mathcal{F}_{t}\right] \leq \frac{1-\delta_{t}^{t+1}}{1-\bar{\delta}}
$$

In the following we will inductively construct the set of states in which the strategy that we will employ in the game with stochastic discounting, would prescribe the play to continue following $\bar{f}$. Consider $\mathbf{d}_{0}>\delta_{\nu}$, and recall that it is deterministic. Then, let $\bar{\delta}_{(1)}$ be such that $\bar{\delta}_{(1)} \geq \delta_{\nu}$ and

$$
\frac{1-\mathbf{d}_{0}}{1-\bar{\delta}_{(1)}} \leq p_{\nu}
$$

and define

$$
\Omega_{(1)}^{\nu}=\left\{\delta \in \Omega: \delta>\delta_{\nu} \text { and } \frac{1-\delta}{1-\bar{\delta}_{(1)}} \leq p_{\nu}\right\} .
$$

Now, given $\bar{\delta}_{(t-1)}$ and $\Omega_{(t-1)}^{\nu}$, define $\bar{\delta}_{(t)}$ such that $\bar{\delta}_{(t)} \geq \delta_{\nu}$ and for any $\delta \in \Omega_{(t-1)}^{\nu}$,

$$
\frac{1-\delta}{1-\bar{\delta}_{(t)}} \leq p_{\nu}
$$

\footnotetext{
${ }^{11}$ Doob's Maximal Inequality for nonnegative submartingales is as follows: Let $\left\{\mathbf{X}_{t}\right\}_{t \in \mathbb{N}_{0}}$ be a nonnegative submartingale with a filtration $\left\{\mathcal{F}_{t}\right\}_{t \in \mathbb{N}_{0}}$ and $\ell>0$. Then for any $T$ for any $s<T$, $\ell \operatorname{Pr}\left[\sup _{s \leq t \leq T} X_{t} \geq \ell \mid \mathcal{F}_{s}\right] \leq \mathrm{E}\left(X_{T} \mid \mathcal{F}_{s}\right)$.
} 
and let

$$
\Omega_{(t)}^{\nu}=\left\{\delta \in \Omega: \delta>\delta_{\nu} \text { and } \frac{1-\delta}{1-\bar{\delta}_{(t)}} \leq p_{\nu}\right\}
$$

Notice that for any history $h$, with $\delta_{t}^{t+1} \in \Omega_{(t)}^{\nu}$, it must be that $\delta_{t}^{t+1}$ is not only strictly above $\delta_{\nu}$, but also, the probability of any one of the future one-shot discount factors being less than or equal to $\bar{\delta}_{(t)}$ is less than or equal to $p_{\nu}$. An important observation is that when $\mathbf{d}_{0}$ is chosen sufficiently high, then $\Omega_{(t)}^{\nu} \neq \emptyset$ for all $t \in \mathbb{N}$. This follows from the denseness of $\Omega^{E}$ (the ergodic set of states) in $\Omega$ following the fourth part of Assumption 2 .

The strategy we use as follows: For any history $h=\left(\bar{h}, d^{t}\right)$ for some $t \in \mathbb{N}_{0}$ with $\ell(h)=\ell(\bar{h})=t$

$$
f(h)= \begin{cases}\bar{f}(\bar{h}) & \text { if } \delta_{s}^{s+1} \in \Omega_{(s)}^{\nu} \text { for all } s \leq t \\ a^{*} & \text { otherwise. }\end{cases}
$$

In words, this strategy prescribes the continuation along the simple strategy $\bar{f}$ whenever the history is one in which the following hold: In any period $t$, all realizations of one-shot discount factors up to period $t, \delta_{s}^{s+1}$ with $s \leq t$, have been such that (1) each one of them is strictly above $\delta_{\nu}$, and (2) the probability evaluated with date $s$ information of any one of the future one-shot discount factors, $\delta_{k}^{k+1}$ with $k \geq s$, being less than or equal to $\bar{\delta}_{(s)}$ is less than or equal to $p_{\nu}, s \leq t$. For all other cases, the strategy prescribes the repetitions of the Nash action profile of the stage game. An interesting observation about this strategy $f$ is that it induces the play to be in only $(n+2)$ states, namely, $\left(\bar{\pi}^{(0)}, \bar{\pi}^{(1)}, \ldots, \bar{\pi}^{(n)}, \pi^{*}\right)$, where $\pi^{*, t}=a^{*}$ for all $t \in \mathbb{N}_{0}$.

Clearly, this strategy is well defined.

Consider any history $h$. Below, we will prove that when $\mathbf{d}_{0}=\hat{\delta}$ is chosen sufficiently high, $f$ is Nash in the subgame starting at $h$, hence, subgame perfect.

If $\delta_{s}^{s+1} \notin \Omega_{(s)}^{\nu}$ for some $s \leq t, f$ recommends the repetition of $a^{*}$ thereafter. Hence, is clearly Nash in such subgames.

If $\delta_{s}^{s+1} \in \Omega_{(s)}^{\nu}$ for all $s \leq t, f$ recommends the continuation of the simple strategy given by $\bar{f}$, which is associated with $\left(\bar{\pi}^{(0)}, \bar{\pi}^{(1)}, \ldots, \bar{\pi}^{(n)}\right)$. Then, the continuation 
utility, equation 2 , can be written as follows:

$$
V_{i}^{t, d^{t}}\left(f,\left\{\mathbf{d}_{t}\right\}_{t}\right)=(1-\hat{\delta}) \sum_{k=t}^{\infty} \mathrm{E}_{t}\left(\delta_{t}^{k}\right)\left(u_{i}\left(\pi^{k}(f)\left(d^{k}\right)\right)\left(1-\rho_{k}^{(t)}\right)+u_{i}\left(a^{*}\right) \rho_{k}^{(t)}\right),
$$

where $\mathbf{d}_{0}=\hat{\delta}$ and for any $k \geq t$,

$$
\rho_{k}^{(t)}=1-\operatorname{Pr}\left[\delta_{s}^{s+1} \in \Omega_{(s)}^{\nu}, \text { for all } s \text { with } t \leq s \leq k \mid \mathcal{F}_{t}\right] \leq p_{\nu}
$$

Notice that, given $h$, hence $a^{t},\left(\pi^{k}(f)\left(d^{k}\right)\right)$ is equal to some $\left(\bar{\pi}^{(j), \kappa}\right)$ for some $j \in N \cup\{0\}$ and $\kappa$, whenever $\delta_{s}^{s+1} \in \Omega_{(s)}^{\nu}$ for all $s$ with $t \leq s \leq k$, an event which happens with probability $1-\rho_{k}^{(t)}$. That is, in such cases the play must be in some phase of $\bar{\pi}^{(j)}$ for some $j \in N \cup\{0\}$.

Observe that for any process satisfying Assumption 2 (specifically the Markov property) with $\mathbf{d}_{0}=\hat{\delta}>\delta_{\nu}$, condition $\underline{6}$ directly implies (recall that the history is such that $\delta_{t}^{t+1}>\delta_{\nu}$ for all $t$, and $\left.\mathrm{E}_{t}\left(\delta_{k}^{k+1}\right)=\delta_{t}^{t+1}, k \geq t\right)$

$$
\left\|\sum_{k=t}^{\infty} \mathrm{E}_{t}\left(\delta_{t}^{k}\right)\left(u_{i}\left(\bar{\pi}^{k}(\bar{f})\right)\right)-\sum_{k=t}^{\infty}\left(\delta_{t}^{t+1}\right)^{k}\left(u_{i}\left(\bar{\pi}^{k}(\bar{f})\right)\right)\right\|<\frac{\nu}{6} .
$$

Similarly due to the same reasons, condition 7 implies that

$$
\left\|\sum_{k=t}^{\infty} \mathrm{E}_{t}\left(\delta_{t}^{k}\right)\left(u_{i}\left(a^{*}\right)\right)-\sum_{k=t}^{\infty}\left(\delta_{t}^{t+1}\right)^{k}\left(u_{i}\left(a^{*}\right)\right)\right\|<\frac{\nu}{6} .
$$

Conditions 6 and 7 together with the fact that $\delta_{t}^{t+1}>\delta_{\nu}$ bring about

$$
\begin{aligned}
\frac{\nu}{3}> & \| \delta_{t}^{t+1}\left(\left(1-p_{\nu}\right) \bar{V}_{i}^{t+1}\left(\bar{\pi}^{(j)}, \delta_{t}^{t+1}\right)+p_{\nu} \bar{V}_{i}^{t+1}\left(a^{*}, \delta_{t}^{t+1}\right)\right) \\
& -\frac{\delta_{t}^{t+1}}{1-\hat{\delta}}\left(\left(1-p_{\nu}\right) \sum_{k=t}^{\infty} \mathrm{E}_{t}\left(\delta_{t}^{k}\right)\left(u_{i}\left(\bar{\pi}^{(j), k}(\bar{f})\right)\right)+p_{\nu} \sum_{k=t}^{\infty} \mathrm{E}_{t}\left(\delta_{t}^{k}\right)\left(u_{i}\left(a^{*}\right)\right)\right) \| .
\end{aligned}
$$


Now, using condition 4 we obtain

$$
\begin{aligned}
\frac{\nu}{2}> & \| \delta_{t}^{t+1}\left(\left(1-p_{\nu}\right) \bar{V}_{i}^{t+1}\left(\bar{\pi}^{(j)}, \delta_{t}^{t+1}\right)+p_{\nu} \bar{V}_{i}^{t+1}\left(a^{*}, \delta_{t}^{t+1}\right)\right) \\
& -\frac{\delta_{t}^{t+1}}{1-\hat{\delta}}\left(\left(1-\rho_{t+1}^{(t)}\right) \sum_{k=t}^{\infty} \mathrm{E}_{t}\left(\delta_{t}^{k}\right)\left(u_{i}\left(\bar{\pi}^{(j), k}(\bar{f})\right)\right)+\rho_{t+1}^{(t)} \sum_{k=t}^{\infty} \mathrm{E}_{t}\left(\delta_{t}^{k}\right)\left(u_{i}\left(a^{*}\right)\right)\right) \| \\
= & \| \delta_{t}^{t+1}\left(\left(1-p_{\nu}\right) \bar{V}_{i}^{t+1}\left(\bar{\pi}^{(j)}, \delta_{t}^{t+1}\right)+p_{\nu} \bar{V}_{i}^{t+1}\left(a^{*}, \delta_{t}^{t+1}\right)\right) \\
& -\delta_{t}^{t+1}\left(\left(1-\rho_{t+1}^{(t)}\right) V_{i}^{t+1, d^{t+1}}\left(f,\left\{\mathbf{d}_{t}\right\}_{t}\right)+\rho_{t+1}^{(t)} V_{i}^{t+1, d^{t+1}}\left(a^{*},\left\{\mathbf{d}_{t}\right\}_{t}\right)\right) \|
\end{aligned}
$$

delivering

$$
\begin{aligned}
\frac{\nu}{2}>\| & (1-\hat{\delta}) u_{i}\left(\bar{\pi}^{(j), t}\right)+\delta_{t}^{t+1}\left(\left(1-p_{\nu}\right) \bar{V}_{i}^{t+1}\left(\bar{\pi}^{(j)}, \delta_{t}^{t+1}\right)+p_{\nu} \bar{V}_{i}^{t+1}\left(a^{*}, \delta_{t}^{t+1}\right)\right) \\
& -V_{i}^{t, d^{t}}\left(f,\left\{\mathbf{d}_{t}\right\}_{t}\right) \|
\end{aligned}
$$

and

$$
\begin{aligned}
\frac{\nu}{2}>\| & (1-\hat{\delta}) \max _{a_{i} \in A_{i}} u_{i}\left(a_{i}, \bar{\pi}_{-i}^{(j), t}\right)+\delta_{t}^{t+1}\left(\left(1-p_{\nu}\right) \bar{V}_{i}^{t+1}\left(\bar{\pi}^{(i)}, \delta_{t}^{t+1}\right)+p_{\nu} \bar{V}_{i}^{t+1}\left(a^{*}, \delta_{t}^{t+1}\right)\right) \\
& -\left((1-\hat{\delta}) \max _{a_{i} \in A_{i}} u_{i}\left(a_{i}, \bar{\pi}_{-i}^{(j), t}\right)\right. \\
& \left.+\delta_{t}^{t+1}\left(\left(1-\rho_{t+1}^{(t)}\right) V_{i}^{t+1, d^{t+1}}\left(f,\left\{\mathbf{d}_{t}\right\}_{t}\right)+\rho_{t+1}^{(t)} V_{i}^{t+1, d^{t+1}}\left(a^{*},\left\{\mathbf{d}_{t}\right\}_{t}\right)\right)\right) \|,
\end{aligned}
$$

where $V_{i}^{t+1, d^{t+1}}\left(f,\left\{\mathbf{d}_{t}\right\}_{t}\right)$ in condition 9 , is the continuation payoff of player $i$ 's punishment path in the stochastic game when $\delta_{t+1}^{t+2} \in \Omega_{(t+1)}^{\nu}$ (otherwise, player $i$ 's deviation is followed by the repetitions of the Nash action).

Conditions 5 , and conditions 8 and 9 together imply that

$$
\begin{aligned}
& V_{i}^{t, d^{t}}\left(f,\left\{\mathbf{d}_{t}\right\}_{t}\right)-\left((1-\hat{\delta}) \max _{a_{i} \in A_{i}} u_{i}\left(a_{i}, \bar{\pi}_{-i}^{(j), t}\right)\right. \\
& \left.\quad+\delta_{t}^{t+1}\left(\left(1-\rho_{t+1}^{(t)}\right) V_{i}^{t+1, d^{t+1}}\left(f,\left\{\mathbf{d}_{t}\right\}_{t}\right)+\rho_{t+1}^{(t)} V_{i}^{t+1, d^{t+1}}\left(a^{*},\left\{\mathbf{d}_{t}\right\}_{t}\right)\right)\right) \\
& \quad>\nu-\frac{\nu}{2}-\frac{\nu}{2}=0,
\end{aligned}
$$

showing that $f$ is Nash in every subgame that starts with $h$ such that $\delta_{s}^{s+1} \in \Omega_{(s)}^{\nu}$ for all $s \leq t$ with $\ell(h)=t$. 
Thus, $f$ is subgame perfect.

Choose $\mathbf{d}_{0}=\hat{\delta}>\delta_{\nu}$ such that $\hat{\delta} \in \Omega_{(1)}^{\nu}$. Then, conditions 4 , and $\underline{6}$ and 7 imply

$$
\left\|V_{i}^{0, d^{0}}\left(f,\left\{\mathbf{d}_{t}\right\}_{t}\right)-(1-\hat{\delta}) \sum_{t=0}^{\infty} \mathrm{E}_{0}\left(\delta_{0}^{t}\right)\left(u_{i}\left(\bar{\pi}^{t}(\bar{f})\right)\right)\right\|<\frac{\nu}{2},
$$

and

$$
\left\|(1-\hat{\delta}) \sum_{t=0}^{\infty} \mathrm{E}_{0}\left(\delta_{0}^{t}\right)\left(u_{i}\left(\bar{\pi}^{t}(\bar{f})\right)\right)-(1-\hat{\delta}) \sum_{t=0}^{\infty} \hat{\delta}^{t}\left(u_{i}\left(\bar{\pi}^{t}(\bar{f})\right)\right)\right\|<\frac{\nu}{6} .
$$

These, in turn, finishes the proof because of the following conclusion

$$
\left\|U\left(f,\left\{\mathbf{d}_{\mathbf{t}}\right\}_{t}\right)-\bar{U}(\bar{f}, \hat{\delta})\right\|=\left\|V_{i}^{0, d^{0}}\left(f,\left\{\mathbf{d}_{t}\right\}_{t}\right)-\bar{U}(\bar{f}, \hat{\delta})\right\|<\frac{4}{6} \nu<\nu<\eta
$$

Now, we are ready to present the proof of our subgame perfect Folk Theorem for repeated games with stochastic discounting:

Proof of Theorem 2. The proof of the Folk Theorem of Fudenberg and Maskin (1991) shows that for any $u \in \mathcal{U}^{0}$, there exists some $\bar{\delta} \in(0,1)$ and a strictly enforceable simple strategy $\bar{f}$ in $\bar{G}(\bar{\delta})$ such that for all $\delta \in(\bar{\delta}, 1), \bar{U}(\bar{f}, \delta)=u$.

This follows from considering conditions $2-4$ and 8 in their proof, which guarantee that each phase of play (which they denote $A$ for the equilibrium, $B_{j}$ for the minmax and $C_{j}$ for the reward phases of $j \in N$ ) they consider satisfies incentive conditions strictly. Additionally, because that each phase of play involves play consisting of cycles, their strategy becomes simple and strictly enforceable when attention is restricted to obtaining individually rational payoffs constructed with the pure strategy minmax.

Hence, Lemma 4 applies and delivers the conclusion that for all $\eta>0$ there exists $\delta^{*} \in(\bar{\delta}, 1)$ such that for all $\hat{\delta}>\delta^{*}$ there is $f$ in $G\left(\left\{\mathbf{d}_{t}\right\}_{t}\right)$ with $\mathbf{d}_{0}=\hat{\delta}$, such that $f$ is subgame perfect in $G\left(\left\{\mathbf{d}_{t}\right\}_{t}\right)$ and $\left\|U\left(f,\left\{\mathbf{d}_{t}\right\}_{t}\right)-\bar{U}(\bar{f}, \hat{\delta})\right\|<\eta$. Thus, letting $\eta \leq \varepsilon$, renders the desired conclusion. 


\section{References}

Abreu, D. (1988): "On the Theory of Infinitely Repeated Games with Discounting," Econometrica, 56, 383-396.

Abreu, D., D. Pearce, and E. Stachetti (1990): "Toward a Theory of Discounted Repeated Games with Imperfect Monitoring," Econometrica, 58(5), 10411063.

Aumann, R., And L. Shapley (1994): "Long-Term Competition - A GameTheoretic Analysis," in Essays in Game Theory in Honor of Michael Maschler, ed. by N. Megiddo. Springer-Verlag, New York.

Barlo, M., G. Carmona, and H. Sabourian (2007): "Bounded Memory with Finite Action Spaces," Sabancı University, Universidade Nova de Lisboa and University of Cambridge.

(2009): “Repeated Games with One - Memory," Journal of Economic Theory, 144, 312-336.

Baye, M., And D. W. Jansen (1996): "Repeated Games with Stochastic Discounting," Economica, 63(252), 531-541.

Doob, J. (1984): Classical Potential Theory and Its Probabilistic Counterpart. Springer-Verlag.

DutTa, P. (1995): "A Folk Theorem for Stochastic Games," Journal of Economic Theory, 66, 1-32.

Fudenberg, D., D. Levine, And E. Maskin (1994): "The Folk Theorem with Imperfect Public Information," Econometrica, 62(5), 997-1039.

FudenberG, D., And E. Maskin (1986): "The Folk Theorem in Repeated Games with Discounting or with Incomplete Information," Econometrica, 54, 533-554. 
(1991): "On the Dispensability of Public Randomization in Discounted Repeated Games," Journal of Economic Theory, 53, 428-438.

Fudenberg, D., and Y. Yamamato (2010): "Repeated Games Where The Payoffs and Monitoring Structure Are Unknown," Econometrica, 78(5), 1673-1710.

Hansen, L., and S. Richard (1987): "The Role of Conditioning Information in Deducing Testable Restrictions Implied by Dynamic Asset Pricing Models," Econometrica, 55, 587-614.

Harrison, J. M., And D. Kreps (1979): "Martingale and Arbitrage in MultiPeriod Securities Markets," Journal of Economic Theory, 20, 381-408.

Hörner, J., And W. Olszewski (2006): "The Folk Theorem for Games with Private Almost-Perfect Monitoring," Econometrica, 74, 1499-1544.

Hörner, J., T. Sugaya, S. Takahashi, and N. Vieille (2009): "Recursive Methods in Discounted Stochastic Games: An Algorithm for delta Approaching 1 and a Folk Theorem," Cowles Foundation for Research in Economics, Yale University.

Kalai, E., And W. Stanford (1988): "Finite Rationality and Interpersonal Complexity in Repeated Games," Econometrica, 56, 397-410.

Karlin, S., And H. M. TAYlor (1975): A First Course in Stochastic Processes. Academic Press, 2 edn.

Mailath, G., And W. Olszewski (2011): "Folk Theorems with bounded recall under (almost) perfect monitoring," Games and Economic Behavior, 71(1), 174192.

Osborne, M., and A. Rubinstein (1994): A Course in Game Theory. Mit Press, Cambridge. 
ÜrgüN, C. (2011): "Stochastic Discounting in Repeated Games: Awaiting the Almost Inevitable," M. A. Thesis, Sabancı University.

Ross, S. A. (1976): "The Arbitrage Theory of Capital Asset Pricing," Journal of Economic Theory, 13, 341-360.

Rubinstein, A. (1982): "Perfect Equilibrium in a Bargaining Model," Econometrica, 50(1), 97-109.

Sabourian, H. (1998): "Repeated Games with $M$-period Bounded Memory (Pure Strategies)," Journal of Mathematical Economics, 30, 1-35.

Williams, D. (1991): Probability with Martingales. Cambridge University Press. 